DAMTP 98-10.

physics/9802012.

\title{
Invariant tensors and Casimir operators for simple compact Lie groups
}

\author{
Arthur J. Mountain* \\ Department of Applied Mathematics and Theoretical Physics, \\ University of Cambridge, Silver Street, \\ Cambridge CB3 9EW, United Kingdom.
}

March 1998

\begin{abstract}
The Casimir operators of a Lie algebra are in one-to-one correspondence with the symmetric invariant tensors of the algebra. There is an infinite family of Casimir operators whose members are expressible in terms of a number of primitive Casimirs equal to the rank of the underlying group. A systematic derivation is presented of a complete set of identities expressing non-primitive symmetric tensors in terms of primitive tensors. Several examples are given including an application to an exceptional Lie algebra.
\end{abstract}

*E-mail : A.J.Mountain@damtp.cam.ac.uk 


\section{Introduction}

The Casimir operators for a simple Lie algebra are constructed from symmetric tensors invariant under the adjoint action of the group. Given a simple compact Lie group of rank $l$, it is known that there are $l$ such primitive symmetric invariant tensors of order $m_{i}, i=$ $1 \ldots l$. The orders $m_{i}$ are in fact also known because of the correspondence of the primitive symmetric invariant tensors with cocycles of order $\left(2 m_{i}-1\right)$ which are representatives of the de Rham cohomology classes of $G$. However, most methods of constructing symmetric invariant tensors give rise naturally to an infinite family of them, of which only the finite number $l$ can be primitive.

In this paper, we consider the set of invariant tensors arising from symmetrized traces of products of Lie algebra basis elements. These correspond to Casimir operators and arise naturally in many physical theories. It is often useful to be able to express this (infinite) family of tensors in terms of the $l$ primitive tensors (see above). We present a method which systematically generates a complete set of identities expressing non-primitive symmetric invariant tensors of this form in terms of primitive tensors. We illustrate our methods for the classical series of Lie algebras and perform explicit calculations for the examples $A_{2}$, $B_{2}$, and $G_{2}$.

\section{Notation and conventions}

In this paper, we will follow the "physical" convention for Lie groups and use hermitian generators $\left\{X_{i}\right\}$ and the normalization $\operatorname{Tr}\left(X_{i} X_{j}\right)=2 \delta_{i j}$. When the generators are assumed to be in matrix form, we take them to be in the defining representation of the algebra. We will assume there is no distinction between upper and lower indices, their position being dictated by notational convenience. We will take $\epsilon_{i_{1} i_{2} \ldots i_{n}}$ to be the standard totally antisymmetric tensor, with $\epsilon_{123 \ldots n}=1$. We will define from this the unit-weight symbol

$$
\epsilon_{i_{1} i_{2} \ldots i_{n}}^{j_{1} j_{2} \ldots j_{n}}=\frac{1}{n !} \epsilon_{i_{1} i_{2} \ldots i_{n}} \epsilon^{j_{1} j_{2} \ldots j_{n}}=\frac{1}{n !} \sum_{\sigma \in S_{n}}(-1)^{\pi(\sigma)} \delta_{i_{1}}^{j_{\sigma(1)}} \ldots \delta_{i_{n}}^{j_{\sigma(n)}},
$$

where $\pi(\sigma)$ is the parity of the permutation $\sigma$.

\section{Invariant symmetric tensors and Casimirs}

Let $\mathcal{G}$ be a simple algebra of rank $l$ with basis $\left\{X_{i}\right\},\left[X_{i}, X_{j}\right]=f_{i j}^{k} X_{k}, i=1 \ldots \operatorname{dim} \mathcal{G}$, and let $G$ be its (compact) associated Lie group. Let $\left\{\omega^{j}\right\}$ be the dual basis in $\mathcal{G}^{*}, \omega^{j}\left(X_{i}\right)=\delta_{i}^{j}$, and consider a $G$-invariant symmetric tensor $h$ of order $m$ 


$$
h=h_{i_{1} \ldots i_{m}} \omega^{i_{1}} \otimes \ldots \otimes \omega^{i_{m}}
$$

The $G$-invariance (ad-invariance) of $h$ means that

$$
\sum_{s=1}^{m} f_{\nu i_{s}}^{\rho} h_{i_{1} \ldots \widehat{i_{s}} \rho i_{s+1} \ldots i_{m}}=0
$$

This is the case for the symmetric tensors $k^{(m)}$ given by the coordinates

$$
k_{i_{1} \ldots i_{m}}^{(m)}=\operatorname{sTr}\left(X_{i_{1}} \ldots X_{i_{m}}\right) \equiv \operatorname{Tr}\left(X_{\left(i_{1} \ldots X_{i_{m}}\right)}\right)
$$

where $\mathrm{s} \operatorname{Tr}$ is the unit-weight symmetric trace, $\operatorname{sTr}\left(X_{i_{1}} \ldots X_{i_{m}}\right)=\frac{1}{m !} \sum_{\sigma \in S_{m}} \operatorname{Tr}\left(X_{i_{\sigma(1)}} \ldots X_{i_{\sigma(m)}}\right)$. Indices inside round brackets $\left(i_{1}, \ldots, i_{m}\right)$ will also be understood as symmetrized with unit weight. In fact [四], a complete set of $l$ primitive (see below) invariant tensors may be constructed in this way by selecting suitable representations.

Let $G$ be compact so that the Killing tensor may be taken as the unit matrix and let $h_{i_{1} \ldots i_{m}}$ be an arbitrary symmetric invariant tensor. Then the order $m$ element in the enveloping algebra $\mathcal{U}(\mathcal{G})$ defined by

$$
\mathcal{C}^{(m)}=h^{i_{1} \ldots i_{m}} X_{i_{1}} \ldots X_{i_{m}}
$$

commutes with all elements of $\mathcal{G}$. This is so because the commutator $\left[X_{\rho}, \mathcal{C}^{(m)}\right]$ may be written as

$$
\left[X_{\rho}, \mathcal{C}^{(m)}\right]=\sum_{s=1}^{m} f_{\rho \nu}^{i_{s}} h^{i_{1} \ldots \widehat{i_{s}} \nu \ldots i_{m}} X_{i_{1}} \ldots X_{i_{m}}=0
$$

which is indeed zero as a result of the invariance condition (2.2). In fact, the only conditions for the $m$-tensor $h$ to generate a Casimir operator $\mathcal{C}^{(m)}$ of $\mathcal{G}$ of order $m$ are its symmetry (non-symmetric indices would allow us to reduce the order $m$ of $\mathcal{C}^{(m)}$ by replacing certain products of generators by commutators) and its invariance (eq. (2.5)); $h$ does not need to be obtained from a symmetric trace (2.3). Thus for any invariant symmetric tensor $h$ of order $m, \mathcal{C}^{(m)}=h^{i_{1} \ldots i_{m}} X_{i_{1}} \ldots X_{i_{m}}$ is a Casimir of $\mathcal{G}$ of the same order $m$.

It is well known [1, 2, 3, 4, 5, 6, 7, 8] that a simple algebra of rank $l$ has $l$ independent (primitive) Casimir-Racah operators of order $m_{1}, \ldots, m_{l}$, the first of them given by the familiar quadratic Casimir [9] operator $k_{i j} X^{i} X^{j}$ obtained from the Killing tensor $\left(m_{1}=2\right)$. Thus there must be (Cayley-Hamilton) relations among the invariant tensors obtained from 
(2.3) for $m \neq m_{l}$ or otherwise one would obtain an arbitrary number of primitive Casimirs. We shall demonstrate this explicitly for several examples.

\section{Relations among symmetric tensors}

The results in this section involve an important result that, for a matrix $A$, we have the relationship

$$
\operatorname{det} A=\exp (\operatorname{Tr} \log A)
$$

This is clearly true for diagonal $A$ and can be seen to be true generally by conjugation of $A$. We apply this to the case $A=\mathbf{1}+\alpha F$, with $\mathbf{1}$ the identity matrix. and $\alpha$ an arbitrary parameter. We can treat the right hand side of (3.1) as a power series in $\alpha$, giving

$$
\begin{aligned}
\operatorname{det}(\mathbf{1}+\alpha F)= & 1-\frac{\alpha^{2}}{2} \operatorname{Tr} F^{2}+\frac{\alpha^{3}}{3} \operatorname{Tr} F^{3}+\alpha^{4}\left[-\frac{1}{4} \operatorname{Tr} F^{4}+\frac{1}{8}\left(\operatorname{Tr} F^{2}\right)^{2}\right] \\
+ & \alpha^{5}\left[\frac{1}{5} \operatorname{Tr} F^{5}-\frac{1}{6} \operatorname{Tr} F^{2} \operatorname{Tr} F^{3}\right] \\
+ & \alpha^{6}\left[-\frac{1}{6} \operatorname{Tr} F^{6}+\frac{1}{18}\left(\operatorname{Tr} F^{3}\right)^{2}+\frac{1}{8} \operatorname{Tr} F^{2} \operatorname{Tr} F^{4}-\frac{1}{48}\left(\operatorname{Tr} F^{2}\right)^{3}\right] \\
+ & \alpha^{7}\left[\frac{1}{7} \operatorname{Tr} F^{7}-\frac{1}{10} \operatorname{Tr} F^{2} \operatorname{Tr} F^{5}-\frac{1}{12} \operatorname{Tr} F^{3} \operatorname{Tr} F^{4}+\frac{1}{24}\left(\operatorname{Tr} F^{2}\right)^{2} \operatorname{Tr} F^{3}\right] \\
+ & \alpha^{8}\left[-\frac{1}{8} \operatorname{Tr} F^{8}+\frac{1}{12} \operatorname{Tr} F^{2} \operatorname{Tr} F^{6}+\frac{1}{15} \operatorname{Tr} F^{3} \operatorname{Tr} F^{5}+\frac{1}{32}\left(\operatorname{Tr} F^{4}\right)^{2}\right. \\
& \left.-\frac{1}{32} \operatorname{Tr} F^{4}\left(\operatorname{Tr} F^{2}\right)^{2}-\frac{1}{36}\left(\operatorname{Tr} F^{3}\right)^{2} \operatorname{Tr} F^{2}+\frac{1}{16.4 !}\left(\operatorname{Tr} F^{2}\right)^{4}\right]+\ldots
\end{aligned}
$$

In the above, $\operatorname{Tr} F^{n}$ is defined as $F_{i_{1} i_{2}} F_{i_{2} i_{3}} \cdots F_{i_{n} i_{1}}$. All the terms to $\mathcal{O}\left(\alpha^{8}\right)$ are given as these are the terms we shall use below. It is easy to calculate the terms in higher powers of $\alpha$. We will now show how to express non-primitive symmetric invariant tensors in terms of primitive tensors.

\section{The algebras $A_{l}$}

We consider a matrix representation of $S U(n)$ with matrix generators $\left\{X_{i}\right\}$. For our purposes we will always work with the fundamental representation so that $X_{i}$ are $n \times n$ matrices. The rank of the group is $l=n-1$ and its Lie algebra is $A_{l}=\mathfrak{s u}(l+1)$. A basis of the symmetric invariant tensors of $A_{l}$ is given by the order $m$ symmetric traces 
$k^{(m)}, m=2,3, \ldots$ from equation (2.3). The algebra $A_{l}$ has $l$ primitive symmetric invariant tensors. A basis of these is given by $k^{(m)}, m=2 \ldots n$.

We now define the matrix $F$ (see equation 3.2) to be a linear combination of the $X_{i}$, introducing a vector $\mathbf{y}$, as $(F)_{a b}=y^{i}\left(X_{i}\right)_{a b}$. As $\left\{X_{i}\right\}$ are $n \times n$ matrices, no terms of $\mathcal{O}\left(\alpha^{m}\right)$ can appear on the right hand side of (3.2) for $m>n$. Thus these terms must vanish identically, giving us one equation at each order in $\alpha$. This gives us one equation for each of the non-primitive tensors of the form (2.3) in terms of primitive tensors.

\section{The algebras $B_{l}$ and $C_{l}$}

The fundamental matrix representations of the algebras $B_{l}=\mathfrak{s o}(2 l+1)$ and $C_{l}=\mathfrak{s p}(2 l)$ are in terms of $(2 l+1) \times(2 l+1)$ and $2 l \times 2 l$ matrices respectively. In their defining representations these groups preserve the euclidean or symplectic metric $\eta$ respectively and thus the generators $X_{i}$ of these algebras satisfy

$$
X_{i} \eta=-\eta X_{i}^{t}
$$

where $i=1, \ldots, l(2 l+1)$. The symmetrised products of an odd number of generators of the orthogonal and symplectic groups also satisfies (3.3); hence, they are a member of their respective algebras. In particular, as the generators of both $B_{l}$ and $C_{l}$ are traceless, we find that the odd-order tensors $k^{(2 m+1)}$ (see eq. 2.3), arising from symmetrized traces, vanish identically. For both $B_{l}$ and $C_{l}$, we have the property that a basis for the symmetric invariant tensors is given by $k^{(m)}$ for $m=2,4,6, \ldots$. Thus for these examples, all odd powers of $\alpha$ in the expansion (3.2) vanish identically. A basis for the primitive symmetric invariant tensors is given by $k^{(m)}, m=2,4,6, \ldots 2 l$. As above, we can write $F_{a b}=y^{i}\left(X_{i}\right)_{a b}$, where $\left\{X_{i}\right\}$ is a basis for the required Lie algebra in the fundamental representation. Substituting this into (3.2), we see that all terms of $\mathcal{O}\left(\alpha^{m}\right)$ must vanish for $m>2 l$. This gives precisely one equation for each of the non-primitive tensors of the form (2.3) in terms of primitive tensors.

\section{The algebras $D_{l}$}

The algebra $D_{l}=\mathfrak{s o}(2 l)$ is the algebra of antisymmetric $(2 l) \times(2 l)$ matrices. The odd-order tensors $k^{(2 m+1)}$ vanish identically using an identical argument to that used above for $B_{l}$. There are $l$ primitive symmetric invariant tensors of which only $(l-1)$ can be expressed in the form (2.3). These are $k^{(m)}, m=2,4,6, \ldots, 2(l-1)$. The final primitive tensor is the Pfaffian, given by 


$$
P f_{i_{1} i_{2} \ldots i_{l}}=\frac{1}{2^{l} l !} \epsilon^{a_{1} a_{2} \ldots a_{2 l}}\left(X_{i_{1}}\right)_{a_{1} a_{2}}\left(X_{i_{2}}\right)_{a_{3} a_{4}} \ldots\left(X_{i_{l}}\right)_{a_{(2 l-1)} a_{2 l}} .
$$

As before, we introduce a vector $\mathbf{y}$ and write $F_{a b}=y_{i}\left(X^{i}\right)_{a b}$. We rewrite the expression (3.2) in the form

$$
\begin{aligned}
\operatorname{det}(\mathbf{1}+\alpha F) & =1+\alpha^{2} \epsilon_{a_{1} a_{2}}^{b_{1} b_{2}} F_{b_{1}}^{a_{1}} F_{b_{2}}^{a_{2}}+\alpha^{4} \epsilon_{a_{1} a_{2} a_{3} a_{4}}^{b_{1} b_{2} b_{3} b_{4}} F_{b_{1}}^{a_{1}} F_{b_{2}}^{a_{2}} F_{b_{3}}^{a_{3}} F_{b_{4}}^{a_{4}}+\ldots \\
& +\alpha^{2 n} \epsilon_{a_{1} a_{2} \ldots a_{2 n}}^{b_{1} b_{2} \ldots b_{2 n}} F_{b_{1}}^{a_{1}} F_{b_{2}}^{a_{2}} \ldots F_{b_{2 n}}^{a_{2 n}}+\ldots
\end{aligned}
$$

The terms of $\mathcal{O}\left(\alpha^{2 m}\right)$ for $m>l$ express the non-primitive symmetric tensors $k^{(2 m)}$ (see equation 2.3) in terms of primitive tensors. For the $\mathcal{O}\left(\alpha^{2 l}\right)$ terms, we must use the identity

$$
\begin{aligned}
\epsilon_{a_{1} a_{2} \ldots a_{2 l}}^{b_{1} b_{2} \ldots b_{2 l}} F_{b_{1}}^{a_{1}} F_{b_{2}}^{a_{2}} \ldots F_{b_{2 l}}^{a_{2 l}} & =\left(\frac{1}{2^{l} l !} \epsilon_{a_{1} a_{2} \ldots a_{2 l}} F_{a_{1} a_{2}} F_{a_{3} a_{4}} \ldots F_{a_{(2 l-1)} a_{2 l}}\right)^{2} \\
& =P f_{\left(i_{1} \ldots i_{l}\right.} P f_{\left.i_{l+1} \ldots i_{2 l}\right)} y^{i_{1}} \ldots y^{i_{2 l}}
\end{aligned}
$$

This expresses the non-primitive tensor $k^{(2 l)}$ in terms of the Pfaffian $P f$ and the $(l-1)$ tensors $k^{(2 m)}, m<l$, which together are the $l$ primitive symmetric tensors of $D_{l}$.

\section{Examples}

Below are given three explicit examples of construction of non-primitive tensors for simple Lie algebras in terms of primitive tensors. Connections are also made to the geometry of the associated Lie groups. In particular, simple compact Lie groups can be expressed as products of spheres of odd dimension, as far as the cohomology is concerned. Decomposing a Lie group into such a product gives the de Rham cohomology as the Betti numbers [10] for a sphere $S^{n}$ are known to be

$$
b^{(0)}=1, \quad b^{(n)}=1 \quad, \quad b^{(k)}=0 \quad \text { otherwise } .
$$

We can also associate with each symmetric invariant tensor $k$ of order $m$ an antisymmetric invariant tensor of order $(2 m-1)$ according to

$$
\Omega_{i_{1} \ldots i_{2 m-2} \sigma}=f_{\left[i_{1} i_{2}\right.}^{j_{1}} \ldots f_{i_{2 m-3} i_{2 m-2}}^{j_{m-1}} k_{\sigma] j_{1} \ldots j_{m-1}} \quad .
$$

If $k$ is primitive, this gives a $(2 m-1)$-form on $G$ representing a Lie algebra cocycle of order $(2 m-1)$. If $k$ is non-primitive, the expression is identically zero [11. 


\section{The algebras $A_{l}$}

The group $S U(l+1)$ has algebra $\mathfrak{s u}(l+1)=A_{l}$. The cohomology of the group manifold is the cohomology of $l$ spheres as $S U(l+1) \rightarrow S^{2 l+1} \times S^{2 l-1} \times \ldots \times S^{3}$. Thus there are $l$ non-trivial cocycles and $l$ corresponding primitive symmetric invariant tensors as in (4.2). There is a basis for the symmetric invariant tensors of $A_{l}$ given by Sudbery [12], consisting of one symmetric tensor $d^{(k)}$ at each order $k=2,3, \ldots$. Given a normalized basis $\left\{\lambda_{i}\right\}$ for $\mathfrak{s u}(l+1)$, we have the multiplication law

$$
\lambda_{i} \lambda_{j}=\frac{2}{l+1} \delta_{i j}+\left(d_{i j k}+i f_{i j k}\right) \lambda_{k} .
$$

The tensors $f$ and $d$ are respectively the totally antisymmetric and symmetric structure constants. The two lowest-order symmetric invariant tensors are $\delta_{i j}$ and $d_{i j k}$. In terms of these, the Sudbery basis for symmetric tensors is

$$
\begin{aligned}
d_{i_{1} i_{2} i_{3}}^{(4)} & =d_{\left(i_{1} i_{2}\right.}{ }^{x} d_{\left.i_{3} i_{4}\right) x}, \\
d_{i_{1} i_{2} i_{3} i_{4} i_{5}} & =d_{\left(i_{1} i_{2}\right.}{ }^{x} d^{x}{ }^{x}{ }^{y} d_{\left.i_{4} i_{5}\right) y}, \\
d_{i_{1} i_{2} i_{3} i_{4} i_{5} i_{6}} & =d_{\left(i_{1} i_{2}\right.}{ }^{x} d^{x}{ }^{x}{ }^{y}{ }^{y}{ }^{y}{ }^{y}{ }_{4}{ }^{z} d_{\left.i_{5} i_{6}\right) z} \quad .
\end{aligned}
$$

We can choose a basis $d^{(k)}, k=2,3, \ldots, l+1$ of the primitive symmetric invariant tensors of $A_{l}$. These will give rise to representatives of the $l$ primitive cocycles of $A_{l}$ via (4.2).

\section{The algebra $A_{2}$}

The fundamental representation of the algebra $A_{2}=\mathfrak{s u}(3)$ is in terms of eight $(3 \times 3)$ hermitian matrix generators $\left\{\lambda_{i}\right\}$. The rank of $A_{2}$ is two so we have two primitive invariant symmetric tensors; these are given by

$$
\frac{1}{2} \operatorname{Tr}\left(\lambda_{i} \lambda_{j}\right)=\delta_{i j} \quad, \quad \frac{1}{2} \mathrm{~s} \operatorname{Tr}\left(\lambda_{i} \lambda_{j} \lambda_{k}\right)=d_{i j k} \quad .
$$

As far as the cohomology is concerned, the group manifold $S U(3)$ can be decomposed into $S^{5} \times S^{3}$ and there are three- and five-cocycles associated with $\delta_{i j}$ and $d_{i j k}$ respectively (see(4.2)). Defining an arbitrary eight-vector $\mathbf{y}$, writing $(F)_{a b}=y^{i}\left(X_{i}\right)_{a b}$ and substituting this into (3.2) gives expressions for $d^{(m)}, m>3$ in terms of the tensors (4.5). Defining $k_{i_{1} \ldots i_{m}}=\operatorname{sTr}\left(X_{i_{1}} \ldots X_{i_{m}}\right)$ as in (2.3), the first few of these are 


$$
\begin{aligned}
k_{i_{1} i_{2} i_{3} i_{4}} & =2 \delta_{\left(i_{1} i_{2}\right.} \delta_{\left.i_{3} i_{4}\right)}, \\
k_{i_{1} i_{2} i_{3} i_{4} i_{5}} & =\frac{10}{3} \delta_{\left(i_{1} i_{2}\right.} d_{\left.i_{3} i_{4} i_{5}\right)}, \\
k_{i_{1} i_{2} i_{3} i_{4} i_{5} i_{6}} & =\frac{4}{3} d_{\left(i_{1} i_{2} i_{3}\right.} d_{\left.i_{4} i_{5} i_{6}\right)}+2 \delta_{\left(i_{1} i_{2}\right.} \delta_{i_{3} i_{4}} \delta_{\left.i_{5} i_{6}\right)}
\end{aligned} .
$$

The first three non-primitive terms in the Sudbery basis for the symmetric invariant tensors of $A_{2}$ can then be computed as

$$
\begin{aligned}
d_{i_{1} i_{2} i_{3} i_{4}}^{(4)} & =d_{\left(i_{1} i_{2}\right.}{ }^{x} d_{\left.i_{3} i_{4}\right) x}=\frac{1}{3} \delta_{\left(i_{1} i_{2}\right.} \delta_{\left.i_{3}\right) i_{4}} \\
d_{i_{1} i_{2} i_{3} i_{4} i_{5}}^{(5)} & =d_{\left(i_{1} i_{2}\right.}{ }^{x} d^{x}{ }^{x}{ }^{y}{ }^{y} d_{\left.i_{4} i_{5}\right) y}=\frac{1}{3} \delta_{\left(i_{1} i_{2}\right.} d_{\left.i_{3} i_{4} i_{5}\right)} \\
d_{i_{1} i_{2} i_{3} i_{4} i_{5} i_{6}} & =d_{\left(i_{1} i_{2}\right.}{ }^{x} d^{x}{ }^{x}{ }^{y}{ }^{y} d^{y}{ }^{y}{ }^{z}{ }^{z} d_{\left.i_{5} i_{6}\right) z}=\frac{2}{15} d_{\left(i_{1} i_{2} i_{3}\right.} d_{\left.i_{4} i_{5} i_{6}\right)}+\frac{1}{15} \delta_{\left(i_{1} i_{2}\right.} \delta_{i_{3} i_{4}} \delta_{\left.i_{5} i_{6}\right)}
\end{aligned}
$$

\section{The algebras $B_{n}$}

The fundamental representation of the algebra $B_{n}=\mathfrak{s o}(2 n+1)$ is in terms of $(2 n+1) \times$ $(2 n+1)$ antisymmetric matrices. We define a basis $\left\{X_{i}\right\}$, normalized as $\operatorname{Tr}\left(X_{i} X_{j}\right)=2 \delta_{i j}$. There will be $n$ primitive symmetric invariant tensors (equal to the rank of the group). The first two of these are $\delta_{i j}=\frac{1}{2} \operatorname{Tr}\left(X_{i} X_{j}\right)$ and the order four totally symmetric tensor [13] given by

$$
\operatorname{Tr}\left(X_{(i} X_{j} X_{k)}\right)=d_{i j k l}^{(4)} X_{l} \quad .
$$

We notice that the tensors $k^{(m)}$ (given by (2.3)) vanish for $m$ odd and we have a basis for the symmetric tensors of $B_{n}$ in analogy with Sudbery's basis for $A_{n}$, i.e.

$$
\begin{aligned}
d_{i_{1} i_{2} i_{3} i_{4} i_{5} i_{6}}^{(6)} & =d_{\left(i_{1} i_{2} i_{3}\right.}{ }^{x} d_{\left.i_{4} i_{5} i_{6}\right) x}, \\
d_{i_{1} i_{2} i_{3} i_{4} i_{5} i_{6} i_{6} i_{i} i_{8}} & =d_{\left(i_{1} i_{2} i_{3}\right.}{ }^{x} d^{x}{ }_{i_{4} i_{5}}{ }^{y} d^{y}{ }_{\left.i_{6} i_{7} i_{8}\right)} \\
& \vdots
\end{aligned} .
$$

The algebra $B_{n}$ will have n primitive symmetric tensors of orders $2,4, \ldots, 2 n$, giving rise to cocycles of orders $3,7, \ldots,(4 n-1)$ via (4.2). 


\section{The algebra $B_{2}$}

The fundamental representation of the algebra $B_{2}=\mathfrak{s o}(5)$ is in terms of $(5 \times 5)$ antisymmetric matrices with a basis $\left\{X_{i}\right\}$, normalized as above. As far as the cohomology is concerned, the group manifold decomposes as $S O(5) \sim S^{3} \times S^{7}$, giving non-trivial three- and sevencocycles. The three-cocycle is associated with the symmetric tensor $\delta_{i j}=\frac{1}{2} \operatorname{Tr}\left(X_{i} X_{j}\right)$ and the seven-cocycle with the order four symmetric tensor $d_{i j k l}^{(4)}=\frac{1}{2} \operatorname{Tr}\left(X_{(i} X_{j} X_{k)} X_{l}\right)$ according to (4.2). As above, we define an arbitrary vector $\mathbf{y}$, write $(F)_{a b}=y^{i}\left(X_{i}\right)_{a b}$ and substitute this into (3.2). The antisymmetry of the algebra elements means that all traces of odd powers of $F$ vanish identically, giving the result at $\mathcal{O}\left(\alpha^{6}\right)$ :

$$
\operatorname{Tr} F^{6}=\frac{3}{4} \operatorname{Tr} F^{2} \operatorname{Tr} F^{4}-\frac{1}{8}\left(\operatorname{Tr} F^{2}\right)^{3},
$$

or equivalently

$$
k_{i_{1} i_{2} i_{3} i_{4} i_{5} i_{6}}^{(6)}=\frac{3}{2} \delta_{\left(i_{1} i_{2}\right.} k_{\left.i_{3} i_{4} i_{5} i_{6}\right)}^{(4)}-\delta_{\left(i_{1} i_{2}\right.} \delta_{i_{3} i_{4}} \delta_{\left.i_{5} i_{6}\right)} .
$$

Expressions for the higher-order symmetric tensors can be obtained easily.

\section{The algebra $G_{2}$}

The algebra $G_{2}$ can be embedded into $\mathfrak{s o}(7)$; the generators of $S O(7)$ decompose into representations $21 \rightarrow \mathbf{7} \oplus \mathbf{1 4}$ under the action of $G_{2}$. We will work with the sevendimensional representation of $G_{2}$ embedded into the fundamental representation of $\mathfrak{s o}(7)$. This has generators $\left\{X_{i}\right\}$, normalized as $\operatorname{Tr}\left(X_{i} X_{j}\right)=2 \delta_{i j}$. As for the orthogonal groups, the terms odd in $F$ in the expansion (3.2) will vanish identically. It is known 13 that there are two primitive symmetric invariant tensors of $G_{2}$, of order two and six, given by the tensors $k^{(2)}$ and $k^{(6)}$ defined in (2.3). We note the result from [14]:

$$
\mathrm{s} \operatorname{Tr}\left(X_{i} X_{j} X_{k} X_{l}\right)=\frac{1}{4} \operatorname{Tr}\left(X_{(i} X_{j}\right) \operatorname{Tr}\left(X_{k} X_{l}\right) .
$$

Using this and the methods described above, we can now write any symmetric invariant tensor of $G_{2}$ in terms of the two primitive symmetric tensors

$$
k_{i_{1} i_{2}}=\operatorname{Tr}\left(X_{i_{1}} X_{i_{2}}\right) \quad, \quad k_{i_{1} i_{2} i_{3} i_{4} i_{5} i_{6}}=\operatorname{sTr}\left(X_{i_{1}} X_{i_{2}} X_{i_{3}} X_{i_{4}} X_{i_{5}} X_{i_{6}}\right)
$$

\section{Conclusion}

A simple Lie group has a number of primitive symmetric invariant tensors equal to the rank of the group. Other symmetric invariant tensors must be expressible in terms of these. 
This is equivalent to expressing non-primitive Casimirs in terms of primitive Casimirs. We have presented a systematic method for doing this which has been illustrated with three examples including one example of an exceptional Lie algebra.

\section{Acknowledgements}

I thank Alan Macfarlane, Jonathan Evans and Niall Mackay for interesting and helpful discussions. I acknowledge the financial assistance of a PPARC studentship. 


\section{References}

[1] B. Gruber and L. O'Raifeartaigh. $s$-theorem and construction of the invariants of the semisimple compact Lie algebras. J. Math. Phys., 5:1796-1804, 1964.

[2] G. Racah, Sulla caratterizzazione delle rappresentazioni irreducibili dei gruppi semisimplici di Lie, Lincei-Rend. Sc. fis. mat. e nat. VIII, 108-112, 1950; Princeton lectures, CERN-61-8 (reprinted in Ergeb. Exact Naturwiss. 37, 28-84, 1965), SpringerVerlag

[3] I.M. Gel'fand, The center of an infinitesimal group ring, Math. Sbornik 26, 103-112, 1950. (English transl.: Los Alamos Sci. Lab. AEC-TR-6133, 1963 )

[4] L. C. Biedenharn. On the representations of the semisimple Lie groups I. J. Math. Phys., 4:436-445, 1963.

[5] A. Klein. Invariant operators of the unimodular group in $n$ dimensions. J. Math. Phys., 4:1283-1284, 1963.

[6] A. M. Perelomov and V. S. Popov. Casimir operators for semisimple groups. Math. USSR-Izvestija, 2:1313-1335, 1968.

[7] S. Okubo and J. Patera. General indices of representations and Casimir invariants. J. Math. Phys., 25:219-227, 1983.

[8] S. Okubo. Modified fourth-order Casimir invariants and indices for simple Lie algebras. J. Math. Phys., 23:8-20, 1982.

[9] H.B.G. Casimir. Proc. Roy. Acad. Amsterdam, 34:844, 1931.

[10] E. Betti. Ann. Mat. Pura Appl., 4:140-158, 1871.

[11] J. A. de Azcárraga, A. J. Macfarlane, A. J. Mountain, and J. C. Pérez Bueno. Invariant tensors for simple groups. Nucl. Phys., B510:657-687, 1998.

[12] A. Sudbery, Ph.D. Thesis, Cambridge Univ., 1970; Computer-friendly d-tensor identities for $S U(n)$ J. Phys. A23(15), L705-L710, 1990

[13] J.A. de Azcárraga and J.C. Pérez Bueno. Higher order simple Lie algebras. Commun. Math. Phys., 184:669-681, 1997.

[14] S. Okubo. Casimir invariants and vector operators in simple and classical Lie algebras. J. Math. Phys., 18(12):2382-2394, 1977. 